Evan L. Thacker, PhD

Barbara McKnight, $\mathrm{PhD}$

Bruce M. Psaty, MD,

$\mathrm{PhD}$

W.T. Longstreth, Jr., $\mathrm{MD}, \mathrm{MPH}$

Colleen M. Sitlani, PhD

Sascha Dublin, MD, PhD

Alice M. Arnold, PhD

Annette L. Fitzpatrick, $\mathrm{PhD}$

Rebecca F. Gottesman, $\mathrm{MD}, \mathrm{PhD}$

Susan R. Heckbert, MD, $\mathrm{PhD}$

Correspondence to

Dr. Thacker:

elt@uab.edu

Supplemental data at www.neurology.org

\section{Atrial fibrillation and cognitive decline}

\section{A longitudinal cohort study}

\section{ABSTRACT}

Objective: We sought to determine whether in the absence of clinical stroke, people with atrial fibrillation experience faster cognitive decline than people without atrial fibrillation.

Methods: We conducted a longitudinal analysis in the Cardiovascular Health Study, a community-based study of 5,888 men and women aged 65 years and older, enrolled in 1989/1990 or 1992/1993. Participants did not have atrial fibrillation or a history of stroke at baseline. Participants were censored when they experienced incident clinical stroke. Incident atrial fibrillation was identified by hospital discharge diagnosis codes and annual study ECGs. The main outcome was rate of decline in mean scores on the 100-point Modified Mini-Mental State Examination (3MSE), administered annually up to 9 times.

Results: Analyses included 5,150 participants, of whom 552 (10.7\%) developed incident atrial fibrillation during a mean of 7 years of follow-up. Mean 3MSE scores declined faster after incident atrial fibrillation compared with no prior atrial fibrillation. For example, the predicted 5-year decline in mean 3MSE score from age 80 to age 85 was -6.4 points (95\% confidence interval [Cl]: $-7.0,-5.9)$ for participants without a history of atrial fibrillation, but was -10.3 points $(95 \% \mathrm{Cl}$ : $-11.8,-8.9)$ for participants experiencing incident atrial fibrillation at age 80 , a 5 -year difference of -3.9 points (95\% Cl: $-5.3,-2.5)$.

Conclusions: In the absence of clinical stroke, people with incident atrial fibrillation are likely to reach thresholds of cognitive impairment or dementia at earlier ages than people with no history of atrial fibrillation. Neurology ${ }^{\circledR}$ 2013;81:119-125

\section{GLOSSARY}

$\mathbf{C I}=$ confidence interval; DSST $=$ Digit Symbol Substitution Test; ICD-9 = International Classification of Diseases, ninth revision; $\mathbf{3 M S E}=$ Modified Mini-Mental State Examination

Atrial fibrillation and cognitive decline are both strongly related to aging. Cognitive decline has profound effects on independence and on the cost and complexity of medical care. Recent research supports atrial fibrillation as a risk factor for incident dementia, independent of clinical stroke. ${ }^{1-3}$ Dementia diagnosis is based on evidence that a person has experienced significant cognitive decline; however, changes in cognition both before and after crossing the dementia threshold are relevant to older adults' functioning and quality of life. The effect of atrial fibrillation on longitudinal cognitive test score trajectories is not well characterized. Previous investigations of atrial fibrillation in relation to cognitive test scores were small or were from cross-sectional studies that could not evaluate the association of atrial fibrillation with longitudinal changes in cognitive function compared with normal aging. ${ }^{4-11}$

The purpose of this report was to examine the association of incident atrial fibrillation with cognitive trajectories using annual cognitive assessments in a large longitudinal study of older adults. We chose to focus on people without clinical stroke because the deleterious effect of stroke on cognition is already well known. We hypothesized that even in the absence of clinical stroke, participants with incident

From the Cardiovascular Health Research Unit (E.L.T., B.M., B.M.P., C.M.S., S.R.H.) and Departments of Epidemiology (E.L.T., B.M.P., W.T. L., S.D., A.L.F., S.R.H.), Biostatistics (B.M., A.M.A.), Medicine (B.M.P., W.T.L., C.M.S.), Health Services (B.M.P.), and Neurology (W.T.L.), University of Washington; Group Health Research Institute (B.M.P., S.D., S.R.H.), Seattle, WA; Department of Neurology (R.F.G.), Johns Hopkins University, Baltimore, MD; and Department of Epidemiology (E.L.T.), University of Alabama at Birmingham, AL.

A preliminary version of this research was presented at the American Heart Association 52nd Cardiovascular Disease Epidemiology and Prevention Conference, San Diego, CA, March 2012.

Go to Neurology.org for full disclosures. Funding information and disclosures deemed relevant by the authors, if any, are provided at the end of the article. 
atrial fibrillation would experience a faster decline in cognitive test scores compared with participants without a history of atrial fibrillation.

METHODS Standard protocol approvals, registrations, and patient consents. Institutional review boards at the University of Washington and each field center approved the study, and participants gave written informed consent.

Design, setting, and participants. The Cardiovascular Health Study is a community-based prospective study of men and women aged 65 years and older. ${ }^{12}$ The cohort included 5,201 participants enrolled in 1989/1990 and 687 participants enrolled in 1992/1993 at field centers in Washington County, MD; Pittsburgh, PA; Forsyth County, NC; and Sacramento County, CA. Participants were invited to return to the field centers for annual visits including cognitive assessments through 1998/1999. They were eligible for this analysis if they did not have atrial fibrillation on the baseline ECG, did not have a history of stroke at baseline, had at least one cognitive assessment obtained at baseline or during follow-up but before incident clinical stroke, and had complete covariate data (figure e-1 on the Neurology ${ }^{\circledR}$ Web site at www.neurology.org). Participants with atrial fibrillation at baseline were excluded because the amount of time since their initial atrial fibrillation onset was unknown, and time since atrial fibrillation was an important variable in our analysis. Participants were censored after they had experienced incident clinical stroke in order to estimate the relationship of atrial fibrillation with cognitive decline in the absence of clinical stroke.

Assessment of atrial fibrillation. Assessment of atrial fibrillation in the Cardiovascular Health Study and validation of the methods have been described. ${ }^{13,14}$ Participants were assessed with 12-lead ECG at baseline and at annual follow-up visits through 1998/1999. The ECGs were interpreted for the presence of atrial fibrillation or atrial flutter at the Cardiovascular Health Study Electrocardiography Reading Center. Also, hospital discharge diagnoses during follow-up were checked for ICD-9 diagnosis codes for atrial fibrillation or atrial flutter (427.3, 427.31, or 427.32). At baseline, participants were considered to have prevalent atrial fibrillation, and were excluded if atrial fibrillation was present on the baseline ECG. During follow-up, participants were considered to have incident atrial fibrillation when atrial fibrillation or atrial flutter was first identified by hospital discharge diagnosis code in any position or by annual study ECG. Once incident atrial fibrillation was identified, participants were considered to have a history of atrial fibrillation through the end of follow-up.

Assessment of covariates. Assessment of baseline and time-varying covariates is described in appendix e-1.

Assessment of cognitive function. Cognitive function assessment in the Cardiovascular Health Study has been described. ${ }^{15,16}$ Cognitive tests included the Modified Mini-Mental State Examination (3MSE), ${ }^{17}$ administered annually up to 9 times from 1990/1991 to 1998/1999, and the Digit Symbol Substitution Test (DSST) ${ }^{18}$ administered annually up to 10 times from $1989 / 1990$ to $1998 / 1999$. The 3MSE is an expanded version of the more frequently used Mini-Mental State Examination. The 3MSE is a general screening tool assessing several aspects of cognition including memory, orientation, calculation, and verbal fluency. Scores on the 3MSE range from zero (worst) to 100 (best). Prior literature suggests using a $3 \mathrm{MSE}$ cutoff of $<78$ points for dementia screening. ${ }^{19}$ The DSST is a subtest of the Wechsler Adult Intelligence Scale. The DSST assesses the ability to quickly match numbers with symbols, which is influenced by aspects of cognition such as information processing speed, visuomotor coordination, and attention. Scores on the DSST range from zero (worst) to 90 (best). In addition, during the 4 annual visit cycles from 1995/1996 to 1998/ 1999, cognitive function was assessed by phone with the Telephone Interview for Cognitive Status ${ }^{20}$ and the Informant Questionnaire on Cognitive Decline in the Elderly ${ }^{21}$ for some participants who did not have in-person cognitive assessments. Missing 3MSE scores were estimated from Telephone Interview for Cognitive Status and Informant Questionnaire on Cognitive Decline in the Elderly scores when available, using equations previously developed with Cardiovascular Health Study data. ${ }^{15}$

Statistical analysis. The statistical analysis was based on longitudinal 3MSE and DSST scores from eligible participants. There were 5,150 participants for analysis of 3MSE trajectories with 34,844 3MSE scores obtained over a mean of 7 years of follow-up, and 5,279 participants for analysis of DSST trajectories with 36,388 DSST scores obtained over a mean of 6 years of follow-up (figure e-1). Scores on 3MSE and DSST were slightly more likely to be missing after incident atrial fibrillation than in participants without atrial fibrillation.

The aim of the statistical analysis was to describe average cognitive trajectories across age, after incident atrial fibrillation was identified compared with no prior atrial fibrillation. We used mixed-effects linear regression models. ${ }^{22}$ Age, incident atrial fibrillation, and years since incident atrial fibrillation was identified were time-varying and were updated at each cognitive assessment. Details regarding the modeling of age and atrial fibrillation variables are given in appendix e-1. Models were adjusted for baseline covariates chosen a priori: birth year, sex, race, years of education through 12th grade, any education beyond 12th grade, former smoking, current smoking, any alcohol use, alcoholic drinks per week, diabetes, hypertension, systolic blood pressure, coronary heart disease, heart failure, and multiplicative interaction terms for the linear age variable with each baseline covariate except birth year. In additional models, we used time-varying covariates to adjust for incident diabetes, hypertension, coronary heart disease, and heart failure. We also repeated the analysis after excluding participants with baseline heart failure and censoring participants at the occurrence of incident heart failure.

For presentation of the results, model-based estimates and mean baseline values of covariates were used to estimate covariate-adjusted mean cognitive score trajectories across age, differences in mean trajectories (after incident atrial fibrillation minus no prior atrial fibrillation), and $95 \%$ confidence intervals (CIs). Statistical analyses were conducted using Stata versions 10-12 (StataCorp, College Station, TX).

RESULTS During a mean of 7 years of follow-up, incident atrial fibrillation was identified in $552(10.7 \%)$ of 5,150 participants included in 3MSE analyses. They were on average older, a larger proportion was male and white, and they had a higher prevalence of diabetes, hypertension, coronary heart disease, and heart failure than those who did not develop incident atrial fibrillation (table 1).

In figure 1, the model-predicted mean 3MSE score trajectory for participants without prior atrial fibrillation (in blue) shows mean scores above 90 points through age 75 , followed by a decline growing steeper with age leading to mean scores below 68 points after age 90 . The predicted trajectories after incident atrial fibrillation identified at ages 70, 75, 80, and 85 (in red) show mean 3MSE scores at the age when incident atrial fibrillation was first identified were similar to mean scores of participants at the same age without prior atrial fibrillation. However, mean scores declined faster in participants after 
Table 1 Baseline characteristics of participants included in 3MSE analyses

\begin{tabular}{|c|c|c|c|}
\hline Characteristic $^{a}$ & $\begin{array}{l}\text { All participants } \\
(n=5,150)\end{array}$ & $\begin{array}{l}\text { Participants without } \\
\text { incident } A F \text { during } \\
\text { follow-up ( } n=4,598 \text { ) }\end{array}$ & $\begin{array}{l}\text { Participants with } \\
\text { incident AF during } \\
\text { follow-up }(n=552)\end{array}$ \\
\hline Age, y, mean (SD) & $73.0(5.4)$ & $72.9(5.3)$ & $74.4(5.8)$ \\
\hline Birth year, mean (SD) & $1917(5.5)$ & $1917(5.4)$ & $1915(5.9)$ \\
\hline Male, \% & 41.2 & 39.9 & 52.0 \\
\hline Black race, \% & 15.2 & 16.0 & 9.2 \\
\hline Years of education through 12th grade, mean (SD) & $11.0(2.0)$ & $11.0(2.0)$ & $11.0(2.0)$ \\
\hline Any education beyond 12 th grade, $\%$ & 43.5 & 43.3 & 45.1 \\
\hline Former smoking, \% & 41.8 & 41.6 & 43.1 \\
\hline Current smoking, \% & 11.7 & 11.9 & 10.5 \\
\hline Any alcohol use, \% & 50.7 & 50.4 & 52.5 \\
\hline Drinks/wk, mean (SD) & $5.0(8.4)$ & $5.0(8.5)$ & $5.1(7.7)$ \\
\hline Diabetes, \% & 14.9 & 14.7 & 16.3 \\
\hline Hypertension, \% & 57.2 & 56.7 & 61.2 \\
\hline Systolic blood pressure, mm Hg, mean (SD) & $136.0(21.5)$ & $135.7(21.4)$ & $138.1(22.6)$ \\
\hline Diastolic blood pressure, mm Hg, mean (SD) & 70.6 (11.3) & 70.7 (11.3) & 69.8 (11.2) \\
\hline Coronary heart disease, \% & 18.2 & 16.9 & 28.6 \\
\hline Heart failure, \% & 3.3 & 3.1 & 5.3 \\
\hline
\end{tabular}

Abbreviations: $\mathrm{AF}=$ atrial fibrillation; 3MSE $=$ Modified Mini-Mental State Examination.

a Values for diastolic blood pressure were missing for 7 participants; means for that variable were calculated among participants without missing values. Mean drinks per week was calculated among participants with any alcohol use.

Figure $1 \quad$ Model-predicted mean 3MSE score trajectories

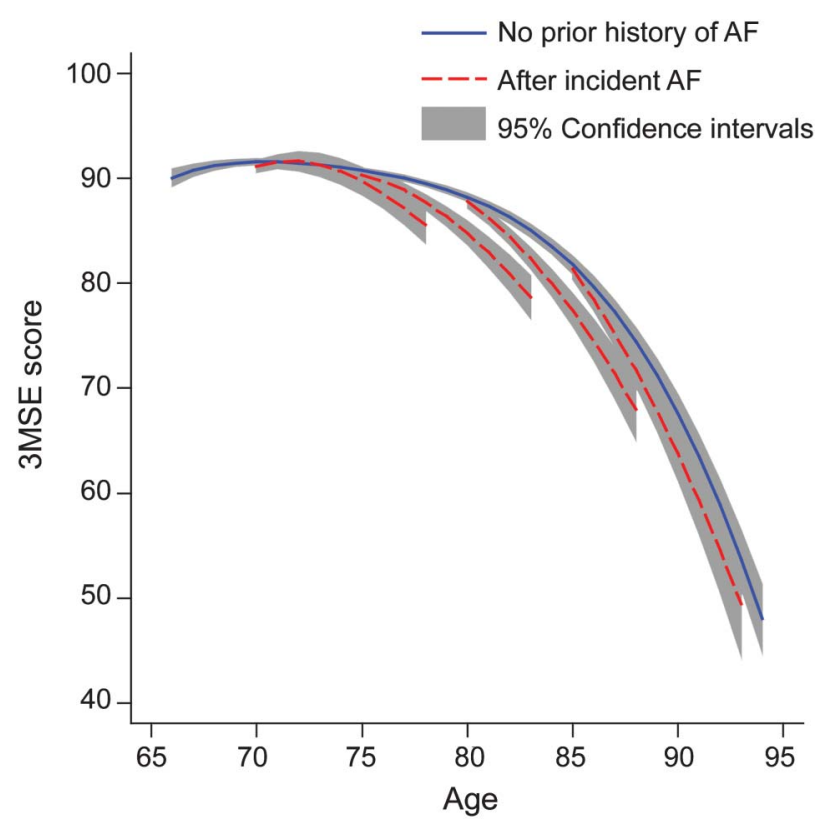

Blue line represents $3 M S E$ trajectory without a history of AF. Four red lines represent $3 M S E$ trajectories after incident AF identified at ages $70,75,80$, and 85 years. Shading represents $95 \%$ confidence intervals. The model included 5,150 participants and was adjusted for birth year, sex, race, education, cigarette smoking, alcohol use, diabetes, hypertension, systolic blood pressure, coronary heart disease, and heart failure, all assessed at baseline, and the interaction of age with each covariate except birth year. AF = atrial fibrillation; 3MSE $=$ Modified Mini-Mental State Examination. incident atrial fibrillation relative to those with no prior atrial fibrillation (see also table e-1).

Model-predicted decline in mean 3MSE score over selected 5-year age periods beginning at ages 70, 75, 80 , and 85 is shown in table 2. For example, with adjustment for baseline covariates, the decline in predicted mean 3MSE score over the next 5 years beginning at age 80 was -6.4 points $(95 \% \mathrm{CI}:-7.0,-5.9)$ for participants without atrial fibrillation who remained free of atrial fibrillation for at least 5 years. However, it was -10.3 points $(95 \% \mathrm{CI}:-11.8,-8.9)$ for participants with incident atrial fibrillation identified at age 80 , a difference of -3.9 points $(95 \% \mathrm{CI}:-5.3,-2.5)$ (model 1 in table 2). Mean 5-year decline in 3MSE score was similarly faster after incident atrial fibrillation identified at ages 75 or 85 , but not at age 70 (model 1 in table 2). Model-predicted trajectories were similar after additional adjustment for other time-varying incident risk factors (model 2 in table 2) and in the absence of heart failure (model 3 in table 2), with some attenuation of the difference between the trajectories after incident atrial fibrillation vs without prior atrial fibrillation.

In figure 2, the model-predicted mean DSST score trajectories after incident atrial fibrillation identified at ages $70,75,80$, and 85 (in red) show faster decline than the trajectory without prior atrial fibrillation (in blue) (see also table e-2). Across all ages, with adjustment for baseline covariates, the predicted decline in mean DSST score over the next 5 years was 2 points greater 
Table 2 Model-predicted 5-year decline in mean (95\% CI) 3MSE score without prior AF and after incident AF

\section{Current age, $y$}

$\begin{array}{lll}70 & 75 & 85\end{array}$

Model $1^{\mathrm{a}}(\mathrm{n}=5,150)$

No prior $\mathrm{AF}^{\mathrm{b}}$

$-0.8(-1.1,-0.5)-2.6(-2.9,-2.3)-6.4(-7.0,-5.9) \quad-14.2(-15.3,-13.0)$

Incident AF identified at current age ${ }^{b}$

Difference (incident AF minus no AF) ${ }^{c}$

Model $2^{d}(n=5,150)$

No prior $\mathrm{AF}^{\mathrm{b}}$

Incident AF identified at current age ${ }^{\text {b }}$

Difference (incident AF minus no AF) ${ }^{c}$

Model $3^{e}(n=4,940)$

No prior $\mathrm{AF}^{\mathrm{b}}$

Incident AF identified at current age ${ }^{b}$

Difference (incident AF minus no AF) ${ }^{c}$
$-1.4(-2.8,0.0)-5.5(-6.7,-4.4)-10.3(-11.8,-8.9)-17.5(-19.7,-15.3)$

$-0.6(-2.1,0.8) \quad-3.0(-4.1,-1.8)-3.9(-5.3,-2.5) \quad-3.4(-5.5,-1.2)$

$-0.7(-1.0,-0.4)-2.4(-2.8,-2.1)-6.2(-6.8,-5.6) \quad-13.7(-14.9,-12.5)$

$-1.2(-2.6,0.3) \quad-5.2(-6.4,-4.1)-9.9(-11.3,-8.4) \quad-16.8(-19.1,-14.6)$

$-0.5(-1.9,1.0) \quad-2.8(-3.9,-1.6)-3.7(-5.1,-2.2) \quad-3.1(-5.3,-0.9)$

$-0.6(-0.9,-0.3)-2.5(-2.8,-2.1)-6.3(-6.9,-5.7) \quad-13.8(-15.0,-12.6)$

$-0.1(-1.8,1.6)-4.7(-6.1,-3.3)-9.5(-11.3,-7.8)-16.4(-19.2,-13.6)$

$0.5(-1.2,2.2) \quad-2.2(-3.6,-0.8)-3.2(-5.0,-1.5) \quad-2.6(-5.3,0.2)$

Abbreviations: $\mathrm{AF}=$ atrial fibrillation; $\mathrm{Cl}=$ confidence interval; 3MSE = Modified Mini-Mental State Examination.

a Model 1: adjusted for birth year, sex, race, education, cigarette smoking, alcohol use, diabetes, hypertension, systolic blood pressure, coronary heart disease, heart failure, and the interaction of age with each covariate except birth year. All covariates were assessed at baseline.

${ }^{\mathrm{b}}$ Larger negative numbers indicate faster decline in cognitive performance.

${ }^{c}$ Larger negative numbers indicate faster decline in cognitive performance after incident AF relative to no prior AF.

${ }^{\mathrm{d}}$ Model 2: adjusted as in model 1, except incident diabetes, hypertension, coronary heart disease, and heart failure were time-varying covariates.

e Model 3: adjusted as in model 2, except participants with heart failure at baseline were excluded, and participants were censored at development of incident heart failure.

\section{Figure 2 Model-predicted mean DSST score trajectories}

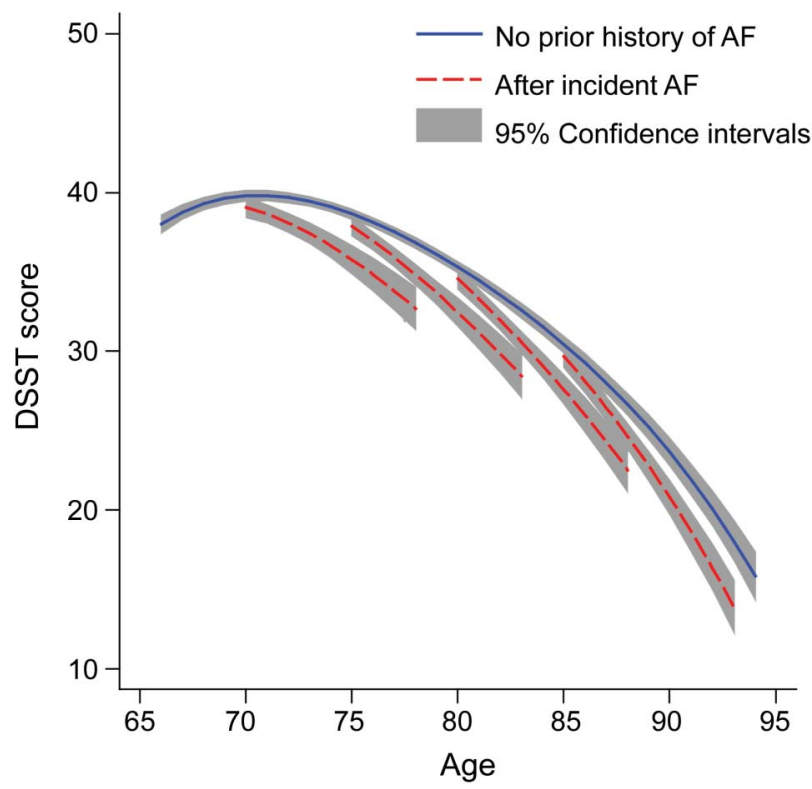

Blue line represents DSST trajectory without a history of AF. Four red lines represent DSST trajectories after incident AF identified at ages 70, 75, 80, and 85 years. Shading represents $95 \%$ confidence intervals. The model included 5,279 participants and was adjusted for birth year, sex, race, education, cigarette smoking, alcohol use, diabetes, hypertension, systolic blood pressure, coronary heart disease, and heart failure, all assessed at baseline, and the interaction of age with each covariate except birth year. AF = atrial fibrillation; DSST $=$ Digit Symbol Substitution Test. (difference: -2.1 points; 95\% CI: $-3.1,-1.2$ ) after incident atrial fibrillation than in participants with no history of atrial fibrillation who remained free of atrial fibrillation for at least 5 years (model 1 in table 3). The association of incident atrial fibrillation with faster decline in mean DSST score remained after adjustment for other time-varying incident risk factors (model 2 in table 3 ) and in the absence of heart failure (model 3 in table 3).

DISCUSSION In this longitudinal study of older adults without stroke, we analyzed annual cognitive scores, including 3MSE for up to 9 years and DSST for up to 10 years. We compared cognitive trajectories after incident atrial fibrillation with trajectories in participants without prior atrial fibrillation. Cognitive decline was more rapid after incident atrial fibrillation was identified than before it was identified. In models of 3MSE trajectories, for ages 75 and above, the average rate of decline was about 3 points faster per 5 years of aging in participants after incident atrial fibrillation relative to those without prior atrial fibrillation. CIs suggested that in the underlying population, the difference in rates of decline may be 1 to 5 points faster per 5 years. In the DSST models, for ages 70 and above, the average rate of decline was about 2 points faster per 5 years in participants after incident atrial fibrillation relative to those without prior atrial fibrillation. CIs suggested an underlying population 
Table 3 Model-predicted 5-year decline in mean (95\% CI) DSST score without prior AF and after incident AF

\section{Current age, y}

70

75

80

85
Model $1^{\text {a }}(n=5,279)$

No prior $\mathrm{AF}^{\mathrm{b}}$
Incident $A F$ identified at current age ${ }^{\mathrm{b}}$
Difference (incident AF minus no $A F)^{c}$
Model $2^{d}$ ( $n=5,279$ )
No prior $A F^{b}$
Incident $A F$ identified at current age ${ }^{b}$
Difference (incident $A F$ minus no $A F)^{c}$
Model $3^{e}$ ( $n=5,079$ )
No prior $A F^{b}$
Incident $A F$ identified at current age ${ }^{b}$
Difference (incident $A F$ minus no $A F)^{c}$

\begin{tabular}{|c|c|c|c|}
\hline$-1.2(-1.4,-0.9)$ & $-3.3(-3.6,-3.1)$ & $-4.9(-5.2,-4.6)$ & $-6.8(-7.3,-6.3)$ \\
\hline$-3.3(-4.2,-2.3)$ & $-5.5(-6.4,-4.5)$ & $-7.0(-7.9,-6.0)$ & $-8.9(-9.9,-7.9)$ \\
\hline$-2.1(-3.1,-1.2)$ & $-2.1(-3.1,-1.2)$ & $-2.1(-3.1,-1.2)$ & $-2.1(-3.1,-1.2)$ \\
\hline$-1.0(-1.2,-0.8)$ & $-3.1(-3.3,-2.8)$ & $-4.5(-4.9,-4.2)$ & $-6.5(-7.0,-6.0)$ \\
\hline$-3.0(-3.9,-2.1)$ & $-5.1(-6.0,-4.1)$ & $-6.6(-7.5,-5.6)$ & $-8.5(-9.5,-7.5)$ \\
\hline$-2.0(-2.9,-1.1)$ & $-2.0(-2.9,-1.1)$ & $-2.0(-2.9,-1.1)$ & $-2.0(-2.9,-1.1)$ \\
\hline$-1.0(-1.2,-0.8)$ & $-3.0(-3.3,-2.8)$ & $-4.5(-4.8,-4.1)$ & $-6.5(-7.1,-6.0)$ \\
\hline$-2.6(-3.7,-1.5)$ & $-4.6(-5.7,-3.5)$ & $-6.1(-7.2,-4.9)$ & $-8.1(-9.3,-6.9)$ \\
\hline$-1.6(-2.7,-0.5)$ & $-1.6(-2.7,-0.5)$ & $-1.6(-2.7,-0.5)$ & $-1.6(-2.7,-0.5)$ \\
\hline
\end{tabular}

Abbreviations: $\mathrm{AF}=$ atrial fibrillation; $\mathrm{Cl}=$ confidence interval; DSST = Digit Symbol Substitution Test.

a Model 1: adjusted for birth year, sex, race, education, cigarette smoking, alcohol use, diabetes, hypertension, systolic blood pressure, coronary heart disease, heart failure, and the interaction of age with each covariate except birth year. All covariates were assessed at baseline.

${ }^{\mathrm{b}}$ Larger negative numbers indicate faster decline in cognitive performance.

${ }^{c}$ Larger differences indicate faster decline in cognitive performance after incident AF relative to no prior AF.

${ }^{\mathrm{d}}$ Model 2: adjusted as in model 1, except incident diabetes, hypertension, coronary heart disease, and heart failure were time-varying covariates.

${ }^{\text {e }}$ Model 3: adjusted as in model 2, except participants with heart failure at baseline were excluded, and participants were censored at development of incident heart failure.

difference in rates of decline of 1 to 3 points faster per 5 years.

To put these differences in context, we can consider the expected magnitude of decline in cognitive scores among people without atrial fibrillation. In this study, for participants without atrial fibrillation, aging 5 years was associated with average predicted declines in 3MSE score of less than a point at age 70 , about 3 points at age 75,6 points at age 80 , and 14 points at age 85 . The more rapid decline we observed after incident atrial fibrillation suggests that people with atrial fibrillation are likely to reach thresholds of cognitive impairment or dementia at earlier ages than people with no history of atrial fibrillation. In prior research, a 3MSE score $<78$ was found to have $88 \%$ sensitivity and $90 \%$ specificity for detecting dementia. ${ }^{19}$ In the present study, participants without atrial fibrillation had a predicted mean 3MSE score $<78$ points at age 87 . In contrast, participants with incident atrial fibrillation at age 80 had a predicted mean score $<78$ points at age 85,2 years earlier than was predicted for participants aging without a history of atrial fibrillation.

A potential mechanism by which atrial fibrillation could hasten cognitive decline is embolic infarction. ${ }^{23}$ In this study, we excluded participants with prevalent stroke and evaluated cognitive trajectories in the absence of incident clinical stroke, but nonetheless found an association between atrial fibrillation and cognitive decline. However, some participants in our study may have experienced stroke during follow-up without being diagnosed. In the Reasons for Geographic and Racial Differences in Stroke (REGARDS) Study, a history of stroke symptoms in the absence of diagnosed stroke was associated with cognitive impairment. ${ }^{24}$ Also, embolic infarcts may be covert and fail to cause noticeable symptoms but produce lasting tissue damage and functional consequences, which could be a potential explanation for the results we observed. If covert embolic infarction was a mechanism linking atrial fibrillation with cognitive decline, we would expect anticoagulation to reduce cognitive decline. We did not explore the role of anticoagulation in this study because we were concerned that in this observational research setting, cognitive status itself could influence whether people with atrial fibrillation initiate or adhere to anticoagulation treatment. Therefore, differences in 3MSE or DSST trajectories by anticoagulation status could potentially result from the effects of cognition on treatment, rather than the other way around.

Another potential mechanism that could link atrial fibrillation to cognitive decline is cerebral hypoperfusion, ${ }^{23}$ which could occur as a result of reduced cardiac output during atrial fibrillation. That the observed association of atrial fibrillation with cognitive decline was 
attenuated when removing participants with heart failure suggests that low cardiac output due to concomitant heart failure may partially explain the relationship of atrial fibrillation with cognitive decline. Hypoperfusion has been hypothesized to result in leukoaraiosis with associated functional consequences in the brain, ${ }^{25}$ and also to impair the clearance of emboli from cerebral vessels. ${ }^{26}$ The roles of embolic infarction and hypoperfusion in linking atrial fibrillation with cognitive decline could be investigated with serial brain imaging.

In addition to the potential causal mechanisms mentioned above, atrial fibrillation and cognitive decline could be associated if atrial fibrillation was a surrogate marker of other factors affecting cognition. We addressed this possibility by adjusting for demographic, behavioral, and clinical risk factors for cognitive decline. Finally, it is possible that changes in the brain associated with cognitive decline could influence the risk of atrial fibrillation, but this is largely unexplored. ${ }^{27}$

Most research on atrial fibrillation and cognition has focused on the outcome of incident dementia. A 2011 review of this literature reported a meta-analysis hazard ratio of 2.4 (95\% CI: 1.7, 3.5) for atrial fibrillation and incident dementia after clinical stroke, and a meta-analysis hazard ratio of 1.6 (95\% CI: 1.0, 2.7) for atrial fibrillation and incident dementia in studies that included people without stroke. ${ }^{1}$ Significant heterogeneity existed among the studies included in the meta-analysis, and the authors concluded that the association between atrial fibrillation and incident dementia was uncertain, except in patients with stroke. A more recent analysis of atrial fibrillation and incident dementia among people with no history of stroke at baseline from the Adult Changes in Thought Study found a hazard ratio of 1.4 (95\% CI: 1.1, 1.8) for incident dementia associated with atrial fibrillation, in the absence of incident stroke. ${ }^{2}$ Another recent analysis among stroke-free participants in the ONTARGET and TRANSCEND trials found a hazard ratio of 1.2 (95\% CI: 1.0, 1.5) for atrial fibrillation and incident dementia. ${ }^{3}$ The present longitudinal study of cognitive trajectories provides new information about the average rate of cognitive decline associated with atrial fibrillation in the absence of clinical stroke.

One prior study with 3 longitudinal cognitive assessments over 3 years found no association of atrial fibrillation with changes in mean test scores over time, although this may have been because of low power due to a small number of participants. ${ }^{8}$ In the present study, the large number of longitudinal cognitive tests spanning up to a decade per participant, and spanning 3 decades of age across all participants, enabled us to estimate precisely the average cognitive trajectories during aging.

This study has limitations. Ascertainment of atrial fibrillation may have been incomplete and onset dates inaccurate if participants had atrial fibrillation without symptoms, delayed seeking medical care for their symptoms, or were not hospitalized after developing atrial fibrillation. We did not have sufficient information to classify atrial fibrillation according to the frequently used categories of single-episode, paroxysmal, persistent, and permanent. ${ }^{28}$ Instead, we used a simpler classification of after incident atrial fibrillation vs without atrial fibrillation. However, prior research by our group and others has shown that once diagnosed, atrial fibrillation usually recurs and often becomes permanent. ${ }^{29,30} \mathrm{We} \mathrm{did}$ not exclude participants with prevalent atrial fibrillation at baseline if it was not detected by the baseline ECG. Scores on 3MSE and DSST were incomplete. Missing cognitive scores are likely to be lower than observed trajectories would predict, and scores were slightly more likely to be missing after incident atrial fibrillation was identified; therefore, the cognitive decline that actually occurred in the cohort may have been greater than what we observed.

This longitudinal study provides evidence that in the absence of clinical stroke, atrial fibrillation is associated with cognitive decline. Further research is needed to learn how the adverse cognitive consequences of atrial fibrillation may be prevented.

\section{AUTHOR CONTRIBUTIONS}

E.L.T. and B.M.: drafting/revising the manuscript for content, study concept or design, analysis or interpretation of data, statistical analysis. B.M.P.: drafting/revising the manuscript for content, study concept or design, analysis or interpretation of data, acquisition of data, statistical analysis, study supervision or coordination, obtaining funding. W.T.L.: drafting/revising the manuscript for content, study concept or design, analysis or interpretation of data, acquisition of data, study supervision or coordination. C.M.S.: drafting/revising the manuscript for content, study concept or design, analysis or interpretation of data, statistical analysis. S.D.: drafting/revising the manuscript for content, study concept or design, analysis or interpretation of data. A.M.A. and A.L.F.: drafting/revising the manuscript for content, study concept or design, analysis or interpretation of data, contribution of vital reagents/tools/patents, acquisition of data, statistical analysis, study supervision or coordination, obtaining funding. R.F.G.: drafting/revising the manuscript for content, study concept or design, analysis or interpretation of data. S.R.H.: drafting/revising the manuscript for content, study concept or design, analysis or interpretation of data, contribution of vital reagents/tools/patents, acquisition of data, statistical analysis, study supervision or coordination, obtaining funding.

\section{ACKNOWLEDGMENT}

The authors thank the Cardiovascular Health Study participants and staff.

\section{STUDY FUNDING}

This research was supported by contracts HHSN268201200036C, HHSN268200800007C, N01HC55222, N01HC85079, N01HC85080, N01HC85081, N01HC85082, N01HC85083, N01HC85086, and grant HL080295 from the National Heart, Lung, and Blood Institute (NHLBI), with additional contribution from the National Institute of Neurological Disorders and Stroke (NINDS). Additional support was provided by AG023629 from the National Institute on Aging (NIA). A full list of principal CHS investigators and institutions can be found at http://www.chs-nhlbi.org. Dr. Thacker was supported by NHLBI training grant T32HL007902 (PI: David Siscovick). Dr. Dublin was supported by NIA grant K23AG028954. Dr. Heckbert was supported by NHLBI grant HL102214.

\section{DISCLOSURE}

E. Thacker and B. McKnight report no disclosures. B. Psaty is a member of the DSMB for a trial of a device funded by the manufacturer and a member 
of the steering committee for the Yale Open Data Access Project funded by Medtronic. W. Longstreth and C. Sitlani report no disclosures. S. Dublin has received a Merck/American Geriatrics Society New Investigator Award. A. Arnold, A. Fitzpatrick, R. Gottesman, and S. Heckbert report no disclosures. Go to Neurology.org for full disclosures.

Received November 15, 2012. Accepted in final form March 23, 2013.

\section{REFERENCES}

1. Kwok CS, Loke YK, Hale R, Potter JF, Myint PK. Atrial fibrillation and incidence of dementia: a systematic review and meta-analysis. Neurology 2011;76:914-922.

2. Dublin S, Anderson ML, Haneuse SJ, et al. Atrial fibrillation and risk of dementia: a prospective cohort study. J Am Geriatr Soc 2011;59:1369-1375.

3. Marzona I, O'Donnell M, Teo K, et al. Increased risk of cognitive and functional decline in patients with atrial fibrillation: results of the ONTARGET and TRANSCEND studies. CMAJ 2012;184:E329-E336.

4. Elias MF, Sullivan LM, Elias PK, et al. Atrial fibrillation is associated with lower cognitive performance in the Framingham offspring men. J Stroke Cerebrovasc Dis 2006;15:214-222.

5. Jozwiak A, Guzik P, Mathew A, Wykretowicz A, Wysocki H. Association of atrial fibrillation and focal neurologic deficits with impaired cognitive function in hospitalized patients $\geq 65$ years of age. Am J Cardiol 2006;98:1238-1241.

6. Kilander L, Andren B, Nyman H, Lind L, Boberg M, Lithell $\mathrm{H}$. Atrial fibrillation is an independent determinant of low cognitive function: a cross-sectional study in elderly men. Stroke 1998;29:1816-1820.

7. O'Connell JE, Gray CS, French JM, Robertson IH. Atrial fibrillation and cognitive function: case-control study. J Neurol Neurosurg Psychiatry 1998;65:386-389.

8. Park H, Hildreth A, Thomson R, O'Connell J. Non-valvular atrial fibrillation and cognitive decline: a longitudinal cohort study. Age Ageing 2007;36:157-163.

9. Puccio D, Novo G, Baiamonte V, et al. Atrial fibrillation and mild cognitive impairment: what correlation? Minerva Cardioangiol 2009;57:143-150.

10. Sabatini T, Frisoni GB, Barbisoni P, Bellelli G, Rozzini R, Trabucchi M. Atrial fibrillation and cognitive disorders in older people. J Am Geriatr Soc 2000;48:387-390.

11. Wozakowska-Kaplon B, Opolski G, Kosior D, JaskulskaNiedziela E, Maroszynska-Dmoch E, Wlosowicz M. Cognitive disorders in elderly patients with permanent atrial fibrillation. Kardiol Pol 2009;67:487-493.

12. Fried LP, Borhani NO, Enright P, et al. The Cardiovascular Health Study: design and rationale. Ann Epidemiol 1991;1: 263-276.

13. Patton KK, Ellinor PT, Heckbert SR, et al. N-terminal proB-type natriuretic peptide is a major predictor of the development of atrial fibrillation: the Cardiovascular Health Study. Circulation 2009;120:1768-1774.

14. Psaty BM, Manolio TA, Kuller LH, et al. Incidence of and risk factors for atrial fibrillation in older adults. Circulation 1997;96:2455-2461.
15. Arnold AM, Newman AB, Dermond N, Haan M, Fitzpatrick A. Using telephone and informant assessments to estimate missing Modified Mini-Mental State Exam scores and rates of cognitive decline. The Cardiovascular Health Study. Neuroepidemiology 2009;33:55-65.

16. Haan MN, Shemanski L, Jagust WJ, Manolio TA, Kuller L. The role of APOE epsilon4 in modulating effects of other risk factors for cognitive decline in elderly persons. JAMA 1999;282:40-46.

17. Teng EL, Chui HC. The Modified Mini-Mental State (3MS) examination. J Clin Psychiatry 1987;48:314-318.

18. Wechsler D. Wechsler Adult Intelligence Scale-Revised Manual. New York: Psychological Corporation; 1981.

19. Woodford HJ, George J. Cognitive assessment in the elderly: a review of clinical methods. QJM 2007;100:469-484.

20. Brandt J, Spencer M, Folstein M. The telephone interview for cognitive status. Neuropsychiatry Neuropsychol Behav Neurol 1988;1:111-117.

21. Jorm AF, Scott R, Cullen JS, MacKinnon AJ. Performance of the Informant Questionnaire on Cognitive Decline in the Elderly (IQCODE) as a screening test for dementia. Psychol Med 1991;21:785-790.

22. Fitzmaurice GM, Laird NM, Ware JH. Applied Longitudinal Analysis. Hoboken, NJ: John Wiley \& Sons; 2004.

23. Duron E, Hanon O. Vascular risk factors, cognitive decline, and dementia. Vasc Health Risk Manag 2008;4:363-381.

24. Wadley VG, McClure LA, Howard VJ, et al. Cognitive status, stroke symptom reports, and modifiable risk factors among individuals with no diagnosis of stroke or transient ischemic attack in the REasons for Geographic And Racial Differences in Stroke (REGARDS) Study. Stroke 2007;38:1143-1147.

25. O'Sullivan M. Leukoaraiosis. Pract Neurol 2008;8:26-38.

26. Caplan LR, Hennerici M. Impaired clearance of emboli (washout) is an important link between hypoperfusion, embolism, and ischemic stroke. Arch Neurol 1998;55:1475-1482.

27. Taggart P, Critchley H, Lambaise PD. Heart-brain interactions in cardiac arrhythmia. Heart 2011;97:698-708.

28. Fuster V, Ryden LE, Cannom DS, et al. ACC/AHA/ESC 2006 Guidelines for the Management of Patients with Atrial Fibrillation: a report of the American College of Cardiology/American Heart Association Task Force on Practice Guidelines and the European Society of Cardiology Committee for Practice Guidelines (Writing Committee to Revise the 2001 Guidelines for the Management of Patients with Atrial Fibrillation): developed in collaboration with the European Heart Rhythm Association and the Heart Rhythm Society. Circulation 2006;114:e257-e354.

29. Thacker EL, McKnight B, Psaty BM, et al. Association of body mass index, diabetes, hypertension, and blood pressure levels with risk of permanent atrial fibrillation. J Gen Intern Med 2013;28:247-253.

30. Kerr CR, Humphries KH, Talajic M, et al. Progression to chronic atrial fibrillation after the initial diagnosis of paroxysmal atrial fibrillation: results from the Canadian Registry of Atrial Fibrillation. Am Heart J 2005;149:489-496. 